\title{
TOWARDS A SEMIFORMAL DEVELOPMENT METHODOLOGY FOR EMBEDDED SYSTEMS
}

\author{
Lucas Cordeiro $^{1,2}$, Raimundo Barreto ${ }^{1}$ \\ ${ }^{1}$ Departamento de Ciência da Computação - Universidade Federal do Amazonas (DCC/UFAM), Brazil \\ rbarreto@dcc.ufam.edu.br \\ ${ }^{2}$ Centro de Ciências, Tecnologia e Inovação do Pólo Industrial de Manaus (CTPIM), Brazil \\ lucas@ctpim.org.br \\ Meuse Oliveira \\ Centro Federal de Educação Tecnológica de Pernambuco (DAES/CEFET-PE), Brazil \\ mnoj@cin.ufpe.br
}

\begin{abstract}
Keywords: Agile methodologies, Embedded Agile Development, Embedded Software Verification, Platform-Based Design, Real-time Software.

Abstract: In recent days, the amount of functions has increased significantly in embedded products so that systems development methodologies play an important role to ensure the product's quality, cost, and time. Furthermore, this complexity coupled with constantly evolving specifications, has led to propose a semiformal development methodology to support the building of embedded real-time systems. A platform-based design approach has been used to balance costs and time-to-market in relation to performance and functionality constraints. We performed three expressive case studies and we concluded that the proposed methodology significantly reduces design time and improves software modularity and reliability.
\end{abstract}

\section{INTRODUCTION}

Embedded computer systems are used in a wide range of sophisticated systems from mobile phones to Settop boxes providing connectivity alternatives to PCs (personal computers). The amount of functions demanded for these systems has increased so significantly that development time becomes difficult to predict and control. As the system complexity increases, its development lifecycle is also affected. Because of that, system development methodologies must be applied in order to substantially reduce manufacturing and design costs, to manage the product requirements (scope) and timeline, and to meet the system's constrains (e.g., energy consumption, execution time, memory footprint).

Therefore, in practice, current design methodologies are no longer adequate and it is evident that there is a crisis in embedded-software design (Vicentelli, 2002). Several development methodologies that are used successfully to produce software that runs on the PCs are not appropriate for developing embedded systems. These devices contain very different characteristics such as dedicated hardware and software, and constraints that are not common to PCs based systems. Furthermore, severe coding errors such as stack/memory overflow, register address access, and timing must be avoided when designing embedded software (ESW). In order to get rid of such errors, system designer needs techniques that allow them to exhaustively traverse the reachable state space.

Proofs of properties have been around since the early days of computer science, but academic advancements were routinely ignored by industry. Potentially, one of the main difficulties to apply formal methods in industry is that software engineers consider that formal methods are difficult to understand and use. Furthermore, some situations complicate the application of formal methods in real projects such as the demand for complex systems and the fact that several changes in the user requirements might take place. To alleviate this problem, agile methodologies have been introduced into the industry in the last decade in order to attempt a useful compromise between no process and too much process.

Based on this context, our main aim is to propose a semiformal development methodology in order to balance costs and time-to-market in relation to performance and functionality constraints. This methodology is mainly composed of well-known prac- 
tices from Software Engineering and Agile methods (Scrum and XP) which aim at minimizing the main problems present on the software development context (i.e. requirement volatility and risk management), and by others practices that are needed to achieve hardware and software development (i.e. platformbased design proposed by (Vicentelli, 2002)). In addition to that, formal verification techniques are also used to check all possible computations and to ensure the correctness of the embedded system.

The remainder of this paper is organized as follows: Section 2 summarizes the related works. Section 3 is concerned with describing the proposed semiformal development methodology and its main components. Section 4 shows the application of the proposed methodology to the development of the pulse oximeter, digital soft-starter, and induction motor simulator equipments. Section 5 shows the experimental results of our proposed methodology. Finally, section 6 concludes this paper and identifies the next steps of this research.

\section{RELATED WORKS}

There are some works about agile development methodologies for embedded systems. However, there is an interesting paper that describes the experience of applying Agile approaches to the development of firmware for the Intel Itanium processor family (Greene, 2004). Manhart and Schneider (Manhart and Schneider, 2004) also reported a successful industrial experience when partially adopting agile methods in the production of software for embedded systems. Indeed, they made slight modifications in a well established software development process for the automotive branch by adopting some agile elements in order to adequate their process to new needs as flexibility and high speed software production.

A very interesting paper that describes an experiment of applying agile test techniques to ESW is presented in (Schooenderwoert and Morsicato, 2004). In that paper, the authors focus on the test techniques that were applied to a mobile spectrometer. In another paper, Koss and Langr propose some adaptations of test techniques used in object oriented (OO) programming languages to ESW written in C language (Koss and Langr, 2002). There is also an interesting work that describes the application of Extreme Programming's test driven development to embedded systems featuring custom hardware and software designs (Dowty, 2004).

The conceptual framework proposed by Ronkainen e Abrahamsson, evaluate the possi- bility to use agile development practices in embedded software environment (Ronkainen and Abrahamsson, 2003). Vicentelli and Martin proposed a rigorous methodology that aims to (i) deal with integration problems among intellectual property (IP) creators, semiconductor vendors, and design houses, (ii) consider metrics to measure embedded system design, (iii) work from conception to software implementation, and (iv) favor reuse by identifying requirements for real plug-and-play operation (Vicentelli, 2002).

The hardware/software co-design methodology proposed by Gajski (Gajski et al., 2000) aims to develop embedded systems by formally describing the system's functionalities in an executable language rather than a natural language. The executable specification is refined through the system-design tasks of allocation, partition, and refinement. Estimators are also used in order to explore design alternatives.

From the point of view of system design methodologies, our proposed work aims to: (i) tradeoff flexibility and performance by adopting highly programmable platforms, (ii) adopt processes and practices to develop ESW that is under stringent hardware constraints, (iii) support a software driven hardware development approach through a comprehensive flow from specification to implementation, (iv) make use of the iterative and incremental approach in order to offer clearly an iterative process, and ( $v$ ) provide experimental results of the application of the proposed methodology in several embedded systems domains.

The next section provides an overview of the proposed methodology and its main components (processes, lifecycle, and roles).

\section{PROPOSED DEVELOPMENT METHODOLOGY}

The idea behind our proposed methodology is to design electronic systems by assembling them from predesigned and pre-characterized components instead of using full custom design methods. Therefore, it aims to favor a correct and efficient assembly of components as well as it leads to an IP reuse strategy that facilitates the creation and verification of designs from different sources (Vicentelli, 2002).

In this way, the definition of platform-based design in our proposed methodology is described by (Chateau, 2001) which defines "platform-based design as the creation of a stable microprocessor-based architecture that can be rapidly extended, customized for a range of applications, and delivered to customers for quick deployment". 
With these goals in mind, the proposed methodology aims to define roles and responsibilities and provide processes, lifecycle, practices and tools to be applied in embedded real-time system projects. The next subsections describe these elements that make up our proposed approach.

\subsection{Processes Group Overview}

The proposed methodology contains three different processes groups that should be used during the system development: system platform, product development and management.

The system platform processes group aims to instantiate the platform for a given product. It means that the system designer must choose the system components that will be part of the architecture and API platforms from a platform library. After that, the system designer has still the possibility to customize these platforms in order to meet the application constraints. The customization process is carried out by programming the designer-configurable processors and runtime-reconfigurable logic integrated into the platform. The customization process is carried out by successive refinements in an iterative and incremental way into the proposed methodology.

The product development processes group offers practices to develop the application's components and integrate them into the platform. The functionalities which make up the product are partitioned into either hardware or software elements of the platform. Our partitioning algorithms used to carry out this task take into account the energy consumption, execution time, and memory size of the application's components (Kimura et al., 2007).

Defining formally, for an embedded application which consists of a function set $\mathrm{F}=\left\{f_{1}, f_{2}, \ldots, f_{n}\right\}$, a k-way partitioning aims to find out a cluster set $P^{k}=\left\{p_{1}, p_{2}, \ldots, p_{n}\right\}$ which consists of $\mathrm{k}$ clusters $p_{1}, p_{2}, \ldots, p_{n}$ so that $p_{1} \cup p_{2} \cup \ldots \cup p_{n}=O$, and $P_{i} \cap$ $P_{j}=\phi$ for $\mathrm{i} \leq \mathrm{j}, \mathrm{j} \leq \mathrm{k}, \mathrm{i} \neq \mathrm{j}$. Our partitioning algorithms find a partitioning $P^{k}$, so that the cost determined by an objective function is minimal and a set of constraints is satisfied.

The mechanical design is also part of this processes group, but it is out of the scope of this paper. The product development processes group also aims to apply a system design approach in order to $(i)$ model an embedded system in a high level of abstraction (using UML diagrams - Unified Modeling Language), (ii) automate the generation and verification of ESW, (iii) substantially reduce the state space explosion problem, and (iv) improve the system's coverage by stressing and covering variables and func- tion calls in ESW. The key idea of this approach is to worry not only about the HW/SW boundaries, but also to consider higher levels of abstraction.

As depicted in Figure 1, this system design approach focuses on creating firstly the class, state machines, collaboration and sequence diagrams using UML models. After that, the code could be generated automatically in the language selected by the system designer (e.g., SystemC, Java, and C/C++). As UML models do not cover the full embedded system design spectrum, then the test design techniques presented further in Section 3.5 can be applied in order to (i) help the system designer codify the system functions, (ii) test the correctness and timeliness of the application, and (iii) facilitate the verification activities described in Section 3.6.

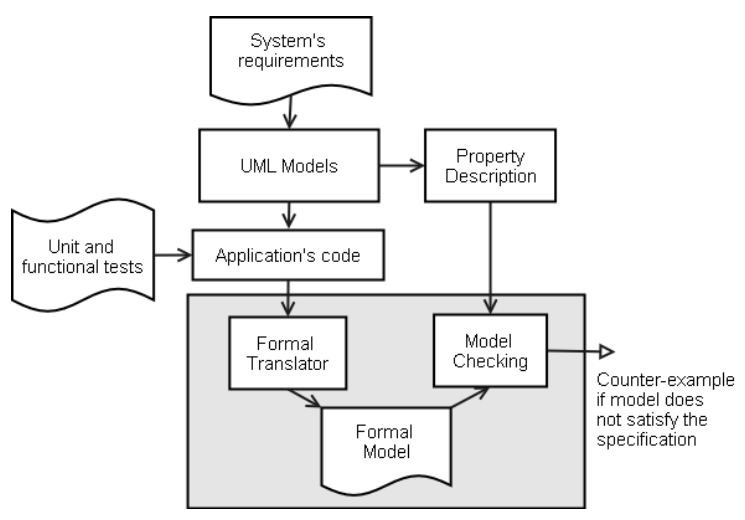

Figure 1: Test Design Approach.

From the application's code, the formal translator converts it to a formal model (e.g., Communicating Finite Automata or Petri Nets) with the purpose of allowing the system designer to verify that certain system's properties hold in the model. The system's properties (e.g., deadlock-freeness, reachability, safety and liveness) are expressed as state formulae such as Linear Temporal Logic (LTL) and Computation Temporal Logic (CTL). Furthermore, if the model does not satisfy the specification then a counter-example is generated which is included into the test suite and after that it is used to test the application's code. The system's properties can be verified using the model checking kit developed by (Esparza et al., 2008).

The product scope, time, quality, and costs parameters are monitored and controlled by the product management processes group. These parameters also influence the system platform and product development processes groups. When the project starts with an infeasible project plan which needs corrective actions to be carried out then this processes group aims to get the project back on the track and to en- 
sure that the project's parameters are met. It is important to emphasize that the product management and development processes groups consist of the practices promoted by the XP and Scrum agile methods as well as the agile patterns described in (Beck and Andres, 2004; Schwaber and Beedle, 2002; Coplien and Schmidt, 2004).

The main reason to propose this semiformal development methodology is to cover the gaps identified by (Abrahamsson et al., 2003; Ronkainen and Abrahamsson, 2003). Therefore, the main motivations of the proposed methodology fall into the following categories: (i) full lifecycle coverage, (ii) project management activities, (iii) flexibility, (iv) means to address the non-functional requirements, (v) a software driven hardware development approach, (vi) concrete guidance of the processes, and (vii) experimental results.

The next subsections are concerned with describing the roles and responsibilities, and the processes lifecycle of the proposed methodology.

\subsection{Roles and Responsibilities}

The proposed methodology involves four different roles and the responsibility of each role is described as follows: Platform Owner is the person who is officially responsible for the products that derive from a given platform. This person is responsible for defining quality, schedule and costs targets of the products. Product leader is responsible for the implementation, integration and test of the product ensuring that quality, schedule, and cost targets defined by the platform owner are met.

Feature Leader is responsible for managing, controlling and coordinating subsystem projects, preintegration projects, external suppliers that contribute to a defined set of features. Development Team which may consist of programmers, architects, and testers are responsible for working on the product development.

If the product to be developed is small, i.e. it is composed of few components (less than 50 KLOC) and does not require other development teams to implement the product's functionalities then one product leader and the development team are enough for the product development. On the other hand, if the product is composed by several components (more than $50 \mathrm{KLOC}$ ) and requires other development teams to implement the product's functionalities then the Feature Leader role must be involved in the processes. In this context, one product leader requires feature leaders to manage, control and coordinate components' projects.

\subsection{Processes Lifecycle}

The proposed agile methodology consists of five phases: Exploration, Planning, Development, Release, and Maintenance. In the Exploration phase, the customers provide requirements for the product releases. These requirements are included into the product backlog by the platform owner in order to estimate the effort with the product leader. In this phase, the development team also identifies the platform and application constraints and estimates the system's metrics based on the product backlog items. With this information at hand, the development team is able to define the system platform.

In the Planning phase, the platform owner and customers identify more requirements and prioritize the product backlog. After that, the development team spends one day to estimate the sprint backlog items and decompose them into tasks. The tasks that make up the sprint backlog must take from 1 to 16 hours to be completed.

In the development phase, the team members implement new functionalities and enhance the system based on the items of the sprint backlog. The meetings are held at the same time and place with the purpose of monitoring and adapting the activities to produce the desired outcomes. At the end of each iteration, unit and functional tests are executed and system properties are checked in a continuous integration build. System optimization also takes place during this phase.

In the Release phase, the product is installed and put into practical use. During this phase, it usually involves the identification of errors and enhancement in the system services. The Maintenance phase may also require more sprints in order to implement new features, enhancement and bug fixes raised in the release phase.

The next subsections describe only three processes of the proposed methodology that focus on achieving the system platform, testing and verifying the system properties of the embedded systems. A detailed description of the thirteen processes of our proposed methodology as well as processes templates are public available for downloading at (Cordeiro, 2008).

\subsection{Process for Instantiating the Platform}

This process would help us estimate the system metrics in order to define the system platform. To obtain the execution time and energy consumption metrics, we could specify the system's functionalities in UML by creating the class, state machines, collaboration 
and sequence diagrams. UML 2.0 profile could also be used to specify the system functionalities (Kukkala et al., 2005), but at the time we wrote this paper there was no tool available to convert the UML 2.0 diagrams into a programming language.

The CASE (Computer Aided Software Engineering) tools like Together and Rational Rose could be used for the entry of the system model. After specifying the system model in UML using these tools, the code could be generated automatically in the language selected by the system designer (e.g., SystemC, Java, and $\mathrm{C} / \mathrm{C}++$ ). Nguyen et. al. (Nguyen et al., 2004) provides a tool that enables the system designer to specify the system model in UML and automatically convert it into SystemC code (Nguyen et al., 2004). After generating the code in the selected language, hardware/software estimation tools could be used to estimate the system metrics. We could use the estimation tool developed by our research group that is capable of estimating the execution time and energy consumption based on Assembly code (Oliveira Jr. et al., 2006).

Therefore, after estimating the system's metrics, we could provide this information to our hardware/software partitioning tool. This tool allows the user to $(i)$ enter the system's model, (ii) enter the objective function parameters such as metrics importance and constraints, (iii) select the components of the system platform, and (iv) find the best partition of the system. Therefore, our tool would look for the best partitioning that meets the design constraints. Since most design decisions are driven by constraints then the application constraints should be incorporated into the objective function so that partitions that meet constraints are considered better than those that do not meet.

\subsection{Process for Implementing New System's Functionalities}

This process would support us for implementing the system's functionalities in a systematic way as well as making the verification steps easier. According to the business value of the system's functionalities, we could start writing the unit test for each stage of computation for those requirements with high business values. However, this kind of activity requires certain level of experience from the system designers. Nonetheless, they should successfully create and compile the unit test before really writing the code and verifying the system's properties.

In order to test each computation stage of the systems' functionalities, we could run the ESW on the PC platform. We could use this approach through- out the development cycle in order to avoid debugging hardware and software simultaneously. By running the ESW on the PC platform, we could exercise all code paths and gain confidence in the code before running it on the target platform. Another way to gain confidence in the code is to use the JTAG debug capability.

We could also create data files on the PC that have all possible parameters combinations that make sense for the system's functions inputs. In this way, we could provide these data to our unit tests to exercise the code's paths of the functions. The unit tests created to the embedded products could be developed by using the embUnit framework test tool (SourceForge, 2007b).

After that, we could run the ESW on the target platform to verify the application's timeliness. It is important to point out that for platform dependent and indepedent code, we could just separate them into different files in order to avoid using the \#if and \#else statements throughout the code.

\subsection{Process for Verifying System's Properties}

The process for verifying the system's properties allows the system designer to check the system's properties by making use of a verification technique called model checking. Therefore, this process aims to exhaustively check all possible computations and observe if a program trace violates temporal and functional properties. If the verification fails, then a path that violates the specification (counter-example) is produced and is later included into the test suite to check the correctness of the system.

However, after converting the application's code into well-formed state machine models, the system designer should then verify if ( $i$ ) no machine has a deadlock, (ii) no machine has nondeterminism behavior, (iii) all outputs and inputs are used. All these checks lead to verifying if local states are reachable to avoid dead code. Therefore, instead of trying to enumerate a design's reachable states, the system designer should first create the state space using Boolean predicates that represent sets of states, and Boolean variables that encode each local machine state. In this way, given a set of states $\mathrm{S}$, the reachability problem could be summarized to determine whether the state $s \in S$ is reachable.

For it to take place, the system designer should determine if $\mathrm{S}$ is reachable by constructing the set of reachable states and then examine whether $S$ intersects this set. The model checking tool that supports this process contributes to practical success because it 
checks from a fixed number of predefined properties rather than requiring the system designer to choose and formalize properties (Esparza et al., 2008). In order to check properties that are specific to the domain being observed, the system designer should then formalize these properties by using logics such as CTL and LTL. Our final aim is to extract the information about the system's properties from the UML diagrams and automatically verify them using the model checking technique.

The next section describes the experimental results of our proposed methodology applied to the domains of medical devices and embedded control systems.

\section{EXPERIMENTAL RESULTS}

In this section we present the results of our proposed methodology applied to the development of the pulse oximeter, digital soft-starter, and the induction motor simulator equipments. The pulse oximeter project was slipt into 3 different sprints and developed by one embedded system engineer. There was also a platform owner who was responsible for defining quality, schedule, costs and requirements of the product. The digital soft-starter and motor simulator projects were split into 2 different sprints and developed by four embedded system engineers (each project had two engineers), one product leader, and one platform owner.

At the beginning of the projects, we created a list of new features and requirements in order to gather all our needs (product backlog). Based on the business value, we chose from the product backlog a set of features and requirements to be implemented in the projects' iterations (sprint backlog). Therefore, we put much emphasis on delivering the systems' functionalities with highest business value in the beginning of the iterations. Delivering these functionalities with highest business value, helped our customer and platform owner get feedback on functionality earlier and allowed them to spot any misunderstanding more quickly. Table 1 shows the measured effort, business value, and sprint velocity for each iteration of the projects.

As can be seen in Table 1, the sprint velocity of the pulse oximeter and soft-starter projects increased as the systems were being developed. This situation took place due to the fact that we were still learning the involved technology, development environment, and the application domain. On the other hand, the sprint velocity of the motor simulator decreased due to the fact that a team member was moved from the soft-starter to another project. Therefore, the tasks
Table 1: Measured Effort (in hours).

\begin{tabular}{rrrr}
\hline & IT1 & IT2 & IT3 \\
\hline \hline Pulse Oximeter & - & - & - \\
Measured Effort & 128 & 127 & 63 \\
Business value & 22 & 27 & 16 \\
Sprint Velocity & 0.17 & 0.21 & 0.25 \\
Digital Soft-Starter & - & - & - \\
Measured Effort & 70 & 84 & - \\
Business value & 11 & 16 & - \\
Sprint Velocity & 0.15 & 0.19 & - \\
Motor Simulator & - & - & - \\
Measured Effort & 125 & 219 & - \\
Business value & 13 & 21 & - \\
Sprint Velocity & 0.10 & 0.09 & - \\
\hline
\end{tabular}

that were allocated to him, had to be transferred to an engineer of the induction motor simulator.

It is important to point out that we chose the pulse oximeter as a case study because it was already developed in another work by our research group using an ad hoc development methodology (Oliveira Jr., 1998). After applying the proposed methodology, the development time was decreased to $50 \%$ when comparing to this ad hoc development methodology. As the proposed methodology does not cover the mechanical design of the product, then the same effort needed by the ad hoc development methodology to design the mechanical part was included into the proposed methodology for comparison reasons.

On our embedded systems projects, we had platform independent code and platform specific code. For platform independent code, we applied our proposed test techniques described in Section 3.5 which check not only the logic but also the timing properties in an automated way.

However, for platform specific code, we had to run it on the target platform manually. For those software classes that brought in sensor data, we just replaced for actual data when running on the PC. That was much better than real data because we had the opportunity to exercise all code paths and to validate the correctness. When running the test cases against these software classes automatically on the PC, we only captured the outputs that would touch the hardware and put them into the log file. Table 2 shows the relationship between the test and code lines of the projects.

The embedded software of our projects had to run in a constrained environment. The development platform used to develop these embedded products had just $12 \mathrm{KBytes}$ of flash memory. Therefore, we used the Big Visible Chart (BVC) proposed by (Beck and Andres, 2004) with the purpose of tracking the 
Table 2: Total LOC (Application and Test).

\begin{tabular}{rrr}
\hline Project & Application & Test \\
\hline \hline Pulse Oximeter & 2685 & 1118 \\
Digital Soft-Starter & 1615 & 854 \\
Motor Simulator & 957 & 243 \\
\hline
\end{tabular}

memory usage and power consumption metrics. Both charts were regularly updated and kept visible in order to look for trends. Table 3 and 4 show the memory usage and power dissipation values of the projects. The current consumption was measured by connecting a multimeter in series with the energy source. The final power dissipation was then obtained by multiplying the current consumption by the supplied voltage. This power is dissipated in the whole system by the digital and analog components.

Table 3: Memory Usage (Bytes).

\begin{tabular}{rrc}
\hline Project & RAM & Flash \\
\hline \hline Pulse Oximeter & 817 & 7711 \\
Digital Soft-Starter & 3631 & 3252 \\
Motor Simulator & 600 & 6398 \\
\hline
\end{tabular}

Table 4: Power Dissipation (mW).

\begin{tabular}{rr}
\hline Project & Power \\
\hline \hline Pulse Oximeter & 414 \\
Digital Soft-Starter & 855 \\
Motor Simulator & 774 \\
\hline
\end{tabular}

The test techniques described in Section 3.5 were the suitable vehicle for software design and modularity of the pulse oximeter, digital soft-stater and motor simulator embedded software. The final solutions of these equipments have approximately 80, 35 and 31 functions in C code respectively. All projects' sprints were analyzed and the result was an average cyclomatic complexity of $3.15,1.43$ and 1.61 at the end of the sprints.

The average cyclomatic complexity $(\mathrm{v}(\mathrm{G}))$ of the systems measures the complexity of a module's decision structure and indicates the number of linearly independent paths. Therefore, these low cyclomatic complexity levels make the white-box testing easier due to the fact that they decrease substantially the number of paths that should be tested to reasonably guard against errors. Data on source code size, number of functions and cyclomatic complexity were obtained using $\mathrm{CCCC}$ tool which analyzes $\mathrm{C} / \mathrm{C}++$ files (SourceForge, 2007a).

The proposed methodology has partially been ap- plied to a multi-site software project in the area of telecommunication containing more than 300 KLOC and involving four different development companies. The results obtained by applying a subset of the processes in this multi-site project can be found at (Cordeiro et al., 2007). The next section concludes this work and provides goals for future research.

\section{CONCLUSIONS AND FUTURE WORK}

This paper described an embedded system development methodology and its application in the development of the pulse oximeter, digital soft-starter and induction motor simulator equipments. In these case studies, the development platform reduced substantially development time of the product. Specifically, for the pulse oximeter case study, we reduced the development time to $50 \%$ when compared to an ad hoc development methodology.

In addition, we also applied a set of test techniques in order to guarantee the timeliness and correctness of the embedded software. These test techniques led to better software design and modularity. Therefore, we obtained $3.15,1.43$, and 1.61 average cyclomatic complexity levels for the pulse oximeter, digital softstarter and motor simulator equipments respectively. For further steps, we are researching models that can carry enough information about the physical implementation and achieve better results in terms of functional correctness.

It is important to point out that development methodologies are very difficult to compare, mainly because they depend on people's pride in their work, wanting to be part of a team, and willingness to pitch in. In order to effectively compare development methodologies, a statistical analysis must be carried out to provide actual results in terms of productivity gains. Therefore, we are planning to perform more experimental studies where the methodology will be observed while applied in different development teams.

\section{ACKNOWLEDGEMENTS}

The authors would like to thank the students Carlos Mar, Eduardo Valentin, Fabiano Teixeira, and Daniel Patrick for the dedication and motivation to accomplish two case studies and also Petrina Kimura for the implementation of the partitioning algorithms. We are very grateful to the support received from the Science 
and Technology Center for the Industrial Pole of Manaus and the Brazilian Council for Scientific and Technological Development (CNPq) by its partial financial support through project 553164/2005-8.

\section{REFERENCES}

Abrahamsson, P., Warsta, J., Siponen, M., and Ronkainen, J. (2003). New directions on agile methods: A comparative analysis. Proceedings of the 25th International Conference on Software Engineering, Portland, Oregon, USA, IEEE Computer Society, pages 244254.

Beck, K. and Andres, C. (2004). Extreme Programming Explained - Embrace Change. Second Edition, AddisonWesley.

Chateau, J. M. (2001). Flexible platform-based design. Online Resources for comms design engineers. Available at http://www.commsdesign.com. Last visit on 14th January 2008.

Coplien, J. O. and Schmidt, D. (2004). Organizational Patterns of Agile Software Development. First Edition, Prentice Hall.

Cordeiro, L. (2008). Txm: Uma metodologia de desenvolvimento de hw/sw para sistemas embarcados. A thesis presented at the Federal University of Amazonas in partial fulfilment of requirements for the degree of Master of Science. Available at http://www.dcc.ufam.edu.br/ lcc/resume.html (In Portuguese) and http://www.dcc.ufam.edu.br/ lcc/methodology (In English).

Cordeiro, L., Becker, C. O., and Barreto, R. S. (2007). Applying scrum and organizational patterns to multi site software development. In 6th Latin American Conference on Pattern Languages of Programming, 2007, Porto de Galinhas, Brazil. SugarLoafPlop'07, pages 46-67.

Dowty, M. (2004). Test driven development of embedded systems using existing software test infrastructure. Available at http://embunit.sourceforge.net/. Last visit on 27th December 2007.

Esparza, J., Schrter, C., and Schwoon, S. (2008). ModelChecking Kit. Published at the University of Stuttgart in the Institute of Formal Methods in Computer Science, Available at http://www.fmi.unistuttgart.de/szs/tools/mckit/. Last visit on 14th January 2008.

Gajski, D., Zhu, J., Dmer, R., Gerstlauer, A., and Zhao, S. (2000). Specc: Specification language and methodology. Kluwer Academic Publishers, Boston, March 2000 .

Greene, B. (2004). Agile methods applied to embedded software development. Proceeding of the Agile Development Conference (ADC'04).

Kimura, P., Barreto, R. S., and Cordeiro, L. C. (2007). Projeto e Implementao de um Plug-in Baseado no
Framework do OSGi para Particionamento de Hardware/Software (In Portuguese). Trabalho de Iniciao Cientfica. Universidade Federal do Amazonas. Conselho Nacional de Desenvolvimento Cientfico e Tecnolgico.

Koss, R. and Langr, J. (2002). Test driven development in c. Available at http://embunit.sourceforge.net/. Last visit on 27th December 2007.

Kukkala, P., Riihimki, J., Hnnikinen, M., Hmlinen, T., and Kronlf, K. (2005). Uml 2.0 profile for embedded system design. Proceedings of the Design, Automation and Test in Europe Conference and Exhibition (DATE'05).

Manhart, P. and Schneider, K. (2004). Breaking the ice for agile development of embedded software: An industry experience report. Proceedings of the 26th International Conference on Software Engineering (ICSE04), page 3647.

Nguyen, K., Sun, Z., , and Thiagarajan, P. (2004). Modeldriven soc design via executable uml to systemc. Proceedings of the 25th IEEE International Symposium on Real-Time Systems, Page(s) 459-468, pages 459468.

Oliveira Jr., M. (1998). Desenvolvimento de Um Protótipo para a Medida Não Invasiva da Saturação Arterial de Oxigênio em Humanos - Oxímetro de Pulso (In Portuguese). Master thesis, Center for Informatics at Federal University of Pernambuco.

Oliveira Jr., M., Neto, S., Maciel, P., Lima, R., Ribeiro, A., Barreto, R., Tavares, E., and Braga, F. (2006). Analyzing software performance and energy consumption of embedded systems by probabilistic modeling: An approach based on coloured petri nets. ICATPN 2006, LNCS 4024, pp. 261281, 2006., page 261281.

Ronkainen, J. and Abrahamsson, P. (2003). Software development under stringent hardware constraints: Do agile methods have a chance? eXtreme Programming Conference.

Schooenderwoert, N. V. and Morsicato, N. (2004). Taming the embedded tiger - agile test techniques for embedded software. Proceedings of the Agile Development Conference (ADC'04).

Schwaber, K. and Beedle, M. (2002). Agile Software Development with Scrum. First Edition, Series in Agile Software Development, Prentice Hall.

SourceForge (2007a). C and $\mathrm{C}++$ Code Counter. Available at http://sourceforge.net/projects/cccc. Last visit on 18 th October.

SourceForge (2007b). embUnit: Unit Test Framework for Embedded C Systems. Available at $\mathrm{http} / / /$ embunit.sourceforge.net/. Last visit on 18th October.

Vicentelli, A. S. (2002). Platform-based design. EEDesign of EETimes. 